\title{
The $\Gamma$-limit for singularly perturbed functionals of Perona-Malik type in arbitrary dimension
}

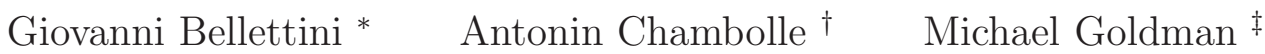

\begin{abstract}
In this paper we generalize to arbitrary dimensions a one-dimensional equicoerciveness and $\Gamma$-convergence result for a second derivative perturbation of Perona-Malik type functionals. Our proof relies on a new density result in the space of special functions of bounded variation with vanishing diffuse gradient part. This provides a direction of investigation to derive approximation for functionals with discontinuities penalized with a "cohesive" energy, that is, whose cost depends on the actual opening of the discontinuity.
\end{abstract}

\section{Introduction}

We investigate in this paper a singular pertubation problem whose limit is defined on piecewise constant functions, and corresponds essentially to a subadditive penalization of the discontinuity.

More precisely, we consider for $\Omega$ a bounded open subset of $\mathbb{R}^{n}$ with Lipschitz boundary, and $\nu \in(0,1]$, the functional $F_{\nu}: L^{2}(\Omega) \rightarrow[0,+\infty]$ defined by

$$
F_{\nu}(u):= \begin{cases}\frac{1}{2} \int_{\Omega}\left[\nu^{3}\left|\nabla^{2} u\right|^{2}+\frac{1}{\nu \phi(1 / \nu)} \phi(|\nabla u|)\right] d x & \text { if } u \in H^{2}(\Omega), \\ +\infty & \text { elsewhere in } L^{2}(\Omega) .\end{cases}
$$

Here $\nabla^{2} u$ is the hessian of $u$ and, for a symmetric real $(n \times n)$-matrix $M$, we set $|M|:=$ $\max \left\{\langle M \xi, \xi\rangle: \xi \in \mathbb{R}^{n},|\xi| \leq 1\right\}$. Moreover the function $\phi: \mathbb{R} \rightarrow[0,+\infty)$ is continuous, even, nondecreasing, typically nonconvex and nonconcave with $\phi^{-1}(0)=\{0\}$, and has sublinear growth at infinity, in the sense that

$$
\exists a \in[0,1) \text { such that } \lim _{p \rightarrow+\infty} \frac{\phi(\lambda p)}{\phi(p)}=\lambda^{a}, \quad \lambda>0 .
$$

\footnotetext{
*Dipartimento di Matematica, Università di Roma Tor Vergata, via della Ricerca Scientifica 1, 00133 Roma, Italy, and LNF-INFN, via E. Fermi 40, 00044 Frascati, Italy. E-mail: belletti@mat.uniroma2.it

${ }^{\dagger}$ CMAP, Ecole Polytechnique, CNRS, Palaiseau, France.

E-mail: antonin.chambolle@cmap.polytechnique.fr

${ }^{\ddagger}$ Max Planck Institute for Mathematics in the Sciences, Leipzig, Germany. E-mail: goldman@mis.mpg.de, partially funded by a Von Humboldt PostDoc fellowship
} 
In this paper we prove that the sequence $\left(F_{\nu}\right)$ is equicoercive and $\Gamma$-converges $[25,16]$ as $\nu \rightarrow 0^{+}$to the functional $\mathcal{F}: L^{2}(\Omega) \rightarrow[0,+\infty]$ defined as follows:

$$
\mathcal{F}(u):= \begin{cases}\sigma_{a} \int_{J_{u}} \mid u^{+}-u^{-\frac{2+a}{4-a}} d \mathcal{H}^{n-1} & \text { if } u \in X(\Omega) \cap L^{2}(\Omega), \\ +\infty & \text { elsewhere in } L^{2}(\Omega),\end{cases}
$$

where $X(\Omega)$ consists of special functions of bounded variation in $\Omega$ having gradient with no absolutely continuous part (see Section 2) and $\sigma_{a}>0$ is a constant depending only on $a$. Our results generalize an equicoerciveness and $\Gamma$-convergence result obtained in [9] in the one-dimensional case.

The interest in these results are twofold. A first important application of such variational problems is in the numerical analysis of fracture mechanics, and in particular the variational approach to fracture growth popularized in the 90's by G. Francfort and J. J. Marigo, built upon the Mumford-Shah functional of image processing [31, 40]. The original model allows to approach the so-called "Griffith" model, where the cost for opening a crack is proportional to its length or area surface. Its success for predicting realistic fractures is impressive [14], however its physical relevance is still a matter of discussion. More physical models (known as "cohesive" and initially introduced by Barenblatt), consider that for a fracture with a small opening, the cost should rather be proportional (through some non-linear correspondence) to the size of the discontinuity. Mathematically, the study of such models is more tricky. Also, finding reasonable approximations of such energies is a difficult problem (still only partially solved, if one really wants to consider linearized or non-linear elasticity energies). Some approaches need to consider a small non-cohesive term $[7,26]$. A more physical term (with slope one when the opening goes to zero) is obtained in [3], which can approach quite general cohesive energies in the scalar setting (and is, to our knowledge, the best and most useful result in this direction so far). It is built as a variant of Ambrosio and Tortorelli's approximation of the Mumford-Shah functional [8]. The variant in [36], built upon finitedifferences approximations, lies in between the previous results since it allows to approach a partially cohesive term with an infinite slope for infinitesimal openings.

This latter result is close to the model that we present here, which is however built upon different ideas. Instead of introducing a phase-field [3] or considering finite differences, we penalize the variations of the gradient by a higher order term. This is similar to the two-wells problem which has been studied in the celebrated paper [21] of Conti, Fonseca, Leoni. Our energy could be considered as a special case, where one well is at 0 and the other at infinity, just as $[13,41]$ are to the standard Modica-Mortola energy ${ }^{1}$.

The second motivation is the relation of our result with the long-time behaviour of solutions to the Perona-Malik equation [42], obtained as the formal $L^{2}$-gradient flow of the functional

$$
\operatorname{PM}(u):=\frac{1}{2} \int_{\Omega} \log \left(1+|\nabla u|^{2}\right) d x,
$$

and corresponding to the choice

$$
\phi(x)=\log \left(1+|x|^{2}\right), \quad x \in \mathbb{R}^{n},
$$

${ }^{1}$ See also $[1,30,22,15]$ for related problems. 
and $a=0$ in (1.2). The Perona-Malik equation therefore reads as

$$
u_{t}=\operatorname{div}\left(\frac{\nabla u}{1+|\nabla u|^{2}}\right)
$$

and it is ill-posed ${ }^{2}$, due to the nonconvexity of $\phi$. In order to overcome the backward parabolic character of (1.4), various regularizations have been suggested in the literature (see [9] and references therein); in particular [27] one can consider, for $\varepsilon \in(0,1]$, the functionals

$$
\operatorname{PM}_{\varepsilon}(u):=\frac{1}{2} \int_{\Omega} \varepsilon^{2}\left|\nabla^{2} u\right|^{2}+\log \left(1+|\nabla u|^{2}\right) d x,
$$

and take the corresponding gradient flow equations, which amounts to add to the equation (1.5) a fourth order term multiplied by $\varepsilon^{2}$; see also [44]. On the basis of the numerical experiments [9] performed when $n=1$, one observes various distinct time scales for the regularized equation; in particular, in a slow time scale, the regularized solutions seem to converge to a piecewise constant function with the plateaus suitably evolving in the vertical direction. The functionals $F_{\nu}$ are related to this slow time scale ${ }^{3}$ setting $\varepsilon^{2}:=\nu^{4} \log \left(1+\frac{1}{\nu^{2}}\right)$

and taking $\frac{\mathrm{PM}_{\varepsilon}}{\nu \log \left(1+\frac{1}{\nu^{2}}\right)}$. Therefore, one expects that the asymptotic limit as $\nu \rightarrow 0^{+}$of the gradient flow of $F_{\nu}$ should shade some light on the behaviour of solutions to (1.5) for large times. This problem has been addressed in [9] when $n=1$. The $n$-dimensional case seems much more difficult, and requires a preliminar study of the asymptotic limit of $F_{\nu}$ and of its variational properties, and this is the content of the present paper. The most original part of this paper concerns the proof of the $\Gamma$-limsup inequality (Theorem 5.5), which relies on a new density result of simple plateaus functions in the space of special functions with bounded variation with vanishing diffuse gradient part, see Lemma 4.1. Differently with respect to the density theorem in [24], our result is valid without assumptions on the measure of the jump set of the limit functions. This allows us to get a full $\Gamma$-convergence result, differently from other related works $[4,39]$, see Remark 5.6.

The plan of the paper is the following. In Section 2 we introduce the notation and recall some definitions about $S B V$ functions and the slicing method. In Section 3 we remind the results of [9] regarding the one-dimensional problem. In Section 4 we state and prove our density result and eventually in Section 5 we prove the equicoerciveness and $\Gamma$-convergence theorems.

\section{Notation}

In what follows $n \geq 1, \Omega \subset \mathbb{R}^{n}$ is a bounded open Lipschitz set, and $\mathcal{A}(\Omega)$ is the class of all open subsets of $\Omega$. We denote by $\left(e_{1}, \ldots, e_{n}\right)$ a fixed orthonormal basis of $\mathbb{R}^{n}$, by $|\cdot|$ (respectively $\langle\cdot, \cdot\rangle)$ the euclidean norm (respectively the euclidean scalar product) in $\mathbb{R}^{n}$. We denote by $\mathcal{H}^{n-1}$ and by $d x$, the $(n-1)$-dimensional Hausdorff measure and the Lebesgue measure in $\mathbb{R}^{n}$. The open ball of radius $\rho>0$ centered at $x \in \Omega$ is denoted by $B_{\rho}(x)$. Throughout the paper, with a small abuse of language, we call sequence a family $\left(u_{\nu}\right)$ of functions labelled by a continuous parameter $\nu \in(0,1]$. A subsequence of $\left(u_{\nu}\right)$ is any sequence $\left(u_{\nu_{k}}\right)$ such that $\nu_{k} \rightarrow 0$ as $k \rightarrow+\infty$.

\footnotetext{
${ }^{2}$ See for instance [38, 37, 33, 35, 11, 10, 12, 28].

${ }^{3}$ We refer also to $[20]$ for the study of the slow time behaviour in a related semi-discrete approximation.
} 


\section{$2.1 \quad B V(\Omega)$ and $S B V(\Omega)$ functions}

$B V(\Omega)$ is the space of functions $u \in L^{1}(\Omega)$ having as distributional derivative $D u$ a measure with finite total variation. For $u \in B V(\Omega)$, we denote by $S_{u}$ the complement of the Lebesgue set of $u$. That is, $x \notin S_{u}$ if and only if $\lim _{\rho \rightarrow 0^{+}} \int_{B_{\rho}(x)}|u(y)-z| d y=0$ for some $z \in \mathbb{R}$. We say that $x$ is an approximate jump point of $u$ if there exist $\xi \in \mathbb{S}^{n-1}$ and distinct $a, b \in \mathbb{R}$ such that

$$
\lim _{\rho \rightarrow 0} \frac{1}{\left|B_{\rho}^{+}(x, \xi)\right|} \int_{B_{\rho}^{+}(x, \xi)}|u(y)-a| d y=0 \quad \text { and } \quad \lim _{\rho \rightarrow 0} \frac{1}{\left|B_{\rho}^{-}(x, \xi)\right|} \int_{B_{\rho}^{-}(x, \xi)}|u(y)-b| d y=0,
$$

where $B_{\rho}^{ \pm}(x, \xi):=\left\{y \in B_{\rho}(x): \pm\langle y-x, \xi\rangle>0\right\}$. Up to a permutation of $a$ and $b$ and a change of sign of $\xi$, this characterize the triplet $(a, b, \xi)$ which is then denoted by $\left(u^{+}, u^{-}, \nu_{u}\right)$. The set of approximated jump points is denoted by $J_{u}$. The following theorem holds [6].

Theorem 2.1. The set $S_{u}$ is countably $\mathcal{H}^{n-1}$-rectifiable and $\mathcal{H}^{n-1}\left(S_{u} \backslash J_{u}\right)=0$. Moreover $D u\left\llcorner J_{u}=\left(u^{+}-u^{-}\right) \nu_{u} \mathcal{H}^{n-1}\left\llcorner J_{u}\right.\right.$.

We indicate by $D u=\nabla u d x+D^{s} u$ the Radon-Nikodym decomposition of $D u$. Setting $D^{c} u:=D^{s} u\left\llcorner\left(\Omega \backslash S_{u}\right)\right.$ we get the decomposition

$$
D u=\nabla u d x+\left(u^{+}-u^{-}\right) \nu_{u} \mathcal{H}^{n-1}\left\llcorner J_{u}+D^{c} u,\right.
$$

where $L$ denotes the restriction. When $n=1$ we use the symbol $u^{\prime}$ in place of $\nabla u$, and $u\left(x^{ \pm}\right)$to indicate the right and left limits at $x$. We let

$$
\begin{aligned}
& S B V(\Omega):=\left\{u \in B V(\Omega): D^{c} u=0\right\}, \\
& G S B V(\Omega):=\left\{u \in L^{1}(\Omega): \max (-T, \min (T, u)) \in S B V(\Omega) \quad \forall T \in \mathbb{R}\right\},
\end{aligned}
$$

and

$$
X(\Omega):=\{u \in S B V(\Omega): \nabla u=0\} .
$$

\subsection{Slicing}

In this section we recall the slicing method for functions with bounded variation. Let $\xi \in \mathbb{S}^{n-1}$ and let

$$
\Pi_{\xi}:=\left\{y \in \mathbb{R}^{n}:\langle y, \xi\rangle=0\right\} .
$$

If $y \in \Pi_{\xi}$ and $E \subset \mathbb{R}^{n}$, we define the one-dimensional slice

$$
E_{\xi y}:=\{t \in \mathbb{R}: y+t \xi \in E\} .
$$

For $u: \Omega \rightarrow \mathbb{R}$, we define $u_{\xi y}: \Omega_{\xi y} \rightarrow \mathbb{R}$ as

$$
u_{\xi y}(t):=u(y+t \xi), \quad t \in \Omega_{\xi y} .
$$

Functions in $G S B V(\Omega)$ can be characterized by one-dimensional slices (see [15, Thm. 4.1]). 
Theorem 2.2. Let $u \in G S B V(\Omega)$. Then for all $\xi \in \mathbb{S}^{n-1}$ we have

$$
u_{\xi y} \in G S B V\left(\Omega_{\xi y}\right) \quad \text { for } \mathcal{H}^{n-1}-\text { a.e. } y \in \Pi_{\xi} \text {. }
$$

Moreover for such $y$, we have

$$
\begin{gathered}
u_{\xi y}^{\prime}(t)=\langle\nabla u(y+t \xi), \xi\rangle \quad \text { for a.e. } t \in \Omega_{\xi y}, \\
J_{u_{\xi y}}=\left\{t \in \mathbb{R}: y+t \xi \in J_{u}\right\}
\end{gathered}
$$

and

$$
u_{\xi y}\left(t^{ \pm}\right)=u^{ \pm}(y+t \xi) \quad \text { or } \quad u_{\xi y}\left(t^{ \pm}\right)=u^{\mp}(y+t \xi),
$$

according to whether $\left\langle\nu_{u}, \xi\right\rangle>0$ or $\left\langle\nu_{u}, \xi\right\rangle<0$. Finally, for every Borel function $g: \Omega \rightarrow \mathbb{R}$,

$$
\int_{\Pi_{\xi}} \sum_{t \in J_{u_{\xi} y}} g_{\xi y}(t) d \mathcal{H}^{n-1}(y)=\int_{J_{u}} g\left|\left\langle\nu_{u}, \xi\right\rangle\right| d \mathcal{H}^{n-1} .
$$

Conversely if $u \in L^{1}(\Omega)$ and if for all $\xi \in\left\{e_{1}, \ldots, e_{n}\right\}$ and almost every $y \in \Pi_{\xi}$ we have $u_{\xi y} \in S B V\left(\Omega_{\xi y}\right)$ and

$$
\int_{\Pi_{\xi}}\left|D u_{\xi y}\right|\left(\Omega_{\xi y}\right) d \mathcal{H}^{n-1}(y)<+\infty
$$

then $u \in S B V(\Omega)$.

\section{The one-dimensional case}

In this section we briefly record the main results of [9], obtained in dimension $n=1$, that will be necessary in order to analyze the problem in arbitrary dimension. For $I \subset \mathbb{R}$ a bounded open interval we consider the functional

$$
F_{\nu}(u, I):= \begin{cases}\frac{1}{2} \int_{I}\left[\nu^{3}\left(u^{\prime \prime}\right)^{2}+\frac{1}{\nu \phi(1 / \nu)} \phi\left(u^{\prime}\right)\right] d x & \text { if } u \in H^{2}(I), \\ +\infty & \text { if } u \in L^{1}(I) \backslash H^{2}(I)\end{cases}
$$

and

$$
\mathcal{F}(u, I):= \begin{cases}\sigma_{a} \sum_{x \in J_{u}}\left|u\left(x^{+}\right)-u\left(x^{-}\right)\right|^{\frac{2+a}{4-a}} & \text { if } u \in X(I), \\ +\infty & \text { if } u \in L^{1}(I) \backslash X(I),\end{cases}
$$

where $X(I)$ is defined, accordingly to the $n$-dimensional case, as $X(I):=\{u \in S B V(I)$ : $\left.u^{\prime}=0\right\}$.

Then the following results hold [9, Lemma 3.2 and Section 4].

Lemma 3.1. There exist $\bar{\nu}>0$, a decreasing function $\omega:(0, \bar{\nu}) \rightarrow(0,+\infty)$ with $\lim _{\nu \rightarrow 0^{+}} \omega(s)=$ 0 , and a constant $C>0$ such that for any $u \in H^{2}(I)$ and $\nu \in(0, \bar{\nu})$,

$$
\int_{I}\left|u^{\prime}\right| d x \leq|I| \omega(\nu)+C F_{\nu}(u, I) .
$$


Theorem 3.2 (Equicoerciveness). Let $\left(u_{\nu}\right) \subset H^{2}(I)$ be a sequence satisfying

$$
\sup _{\nu \in(0,1]} F_{\nu}\left(u_{\nu}, I\right)<+\infty
$$

and such that $\int_{I} u_{\nu} d x=0$ for any $\nu \in(0,1]$. Then there exist a function $u \in X(I)$ and $a$ subsequence of $\left(u_{\nu}\right)$ weakly* converging to $u$ in $B V(I)$.

Theorem 3.3 ( $\Gamma$-convergence). We have

$$
\Gamma\left(L^{1}(I)\right)-\lim _{\nu \rightarrow 0^{+}} F_{\nu}(\cdot, I)=\mathcal{F}(\cdot, I),
$$

and the same result holds true for the $\Gamma\left(L^{2}(I)\right)$-limit.

We also remind the construction of the recovery sequence in the proof of the $\Gamma-\lim$ sup inequality in Theorem 3.3. For any $\eta>0$ and $s \geq 0$, let

$$
Y_{\eta}(s):=\left\{\psi \in H^{2}((0, \eta)): \psi(0)=0, \psi(\eta)=s, \psi^{\prime}(0)=\psi^{\prime}(\eta)=0\right\} .
$$

Define

$$
e_{a}(s):=\frac{1}{2} \inf \left\{\int_{(0, \eta)}\left[\left(\psi^{\prime \prime}\right)^{2}+\left|\psi^{\prime}\right|^{a}\right] d x: \eta>0, \psi \in Y_{\eta}(s)\right\} .
$$

Then it turns out that

$$
e_{a}(s)=\sigma_{a} s^{\frac{2+a}{4-a}}
$$

for a suitable constant $\sigma_{a}>0$.

Fix now $b>0$ and $s>0$. Let $(\eta, \psi) \in(0,+\infty) \times Y_{\eta}(s)$ be such that

$$
\int_{0}^{\eta}\left[\left(\psi^{\prime \prime}\right)^{2}+\left|\psi^{\prime}\right|^{a}\right] d x \leq \sigma_{a} s^{\frac{2+a}{4-a}}+b .
$$

For a function of $X(I)$ of the form $u=s 1_{[0,+\infty)}$, a recovery sequence $\left(u_{\nu}\right) \subset H^{2}(I)$ is given by

$$
u_{\nu}(x):= \begin{cases}0 & x<0 \\ \psi\left(\frac{x}{\nu}\right) & x \in[0, \eta \nu] \\ s & x>\eta \nu\end{cases}
$$

in the sense that for every $\delta>0$ we have that $\left(u_{\nu}\right)$ tends to $u$ in $L^{1}((-\delta, \delta))$ and

$$
\limsup _{\nu \rightarrow 0^{+}} F_{\nu}\left(u_{\nu},(-\delta, \delta)\right)=\sigma_{a} s^{\frac{2+a}{4-a}}+b=\mathcal{F}(u,(-\delta, \delta))+b,
$$

so that in particular $\lim _{b \rightarrow 0^{+}} \limsup _{\nu \rightarrow 0^{+}} F_{\nu}\left(u_{\nu},(-\delta, \delta)\right)=\mathcal{F}(u,(-\delta, \delta))$. A similar construction can be made for $s<0$. 


\section{The $n$-dimensional case: a density result}

Let $\theta: \mathbb{R} \rightarrow \mathbb{R}^{+}$be an even lower semicontinuous function, subadditive and nondecreasing on $(0,+\infty)$, and continuous at $t=0$, such that $\theta(0)=0, \lim _{t \rightarrow 0} \frac{\theta(t)}{t}=+\infty$. Let us notice that the functions $s \rightarrow|s|^{\frac{2+a}{4-a}}$ satisfy these properties.

Define

$$
\mathcal{P}(\Omega):=\{u \in G S B V(\Omega): \nabla u=0 \text { in } \Omega\}
$$

and for any $A \in \mathcal{A}(\Omega)$ set

$$
\mathcal{E}(u, A):= \begin{cases}\int_{J_{u} \cap A} \theta\left(u^{+}-u^{-}\right) d \mathcal{H}^{n-1} & \text { if } u \in \mathcal{P}(A), \\ +\infty & \text { elsewhere in } L^{1}(A) .\end{cases}
$$

We recall that $\mathcal{E}(\cdot, A)$ is $L^{1}(A)$-lower semicontinuous [6, Ex. 5.23].

In this section we want to prove the following result, which is the main technical tool of this paper, and will be used in the proof of Theorem 5.5.

Lemma 4.1 (Density). Let $u \in \mathcal{P}(\Omega)$ be such that $\mathcal{E}(u, \Omega)<+\infty$. Then there exists a sequence $\left(u_{h}\right) \subset \mathcal{P}(\Omega)$ with the following properties:

- $\lim _{h \rightarrow 0^{+}} u_{h}=u$ in $L^{1}(\Omega)$;

- $\lim _{h \rightarrow 0^{+}} \mathcal{E}\left(u_{h}, \Omega\right)=\mathcal{E}(u, \Omega)$;

- $J_{u_{h}}$ is contained in a finite union of facets of polytopes ${ }^{4}$ for any $h \in \mathbb{N}$. In particular for any $h \in \mathbb{N}$,

$$
\mathcal{H}^{n-1}\left(\Omega \cap \bar{J}_{u_{h}}\right)=\mathcal{H}^{n-1}\left(\Omega \cap J_{u_{h}}\right) \quad \text { and } \quad \mathcal{H}^{n-1}\left(\bar{J}_{u_{h}}\right)<+\infty .
$$

Proof. Let $\Omega^{\prime} \supset \supset \Omega$ be an open set and let us denote still by $u \in \mathcal{P}\left(\Omega^{\prime}\right)$ an extension of $u$ such that $\mathcal{H}^{n-1}\left(J_{u} \cap \partial \Omega\right)=0$ (see [32]). We also fix a representative of $u$ defined everywhere. We will need an auxiliary functional $\mathcal{E}_{0}$ defined as follows: for any open set $A \subseteq \Omega^{\prime}$

$$
\mathcal{E}_{0}(v, A):= \begin{cases}\int_{J_{v} \cap A}\left\|\nu_{v}\right\|_{1} \theta\left(v^{+}-v^{-}\right) d \mathcal{H}^{n-1} & \text { if } v \in \mathcal{P}(A), \\ +\infty & \text { elsewhere in } L^{1}(A),\end{cases}
$$

where, for $\xi=\left(\xi_{1}, \ldots, \xi_{n}\right) \in \mathbb{R}^{n}$, we let

$$
\|\xi\|_{1}:=\left|\xi_{1}\right|+\cdots+\left|\xi_{n}\right| .
$$

Again by [6, Ex. 5.23], the functional $\mathcal{E}_{0}(\cdot, A)$ is $L^{1}(A)$-lower semicontinuous.

\footnotetext{
${ }^{4} \mathrm{~A}$ polytope is a set whose boundary consists of a finite number of pieces of hyperplanes.
} 
Observe that $\nu \in \mathbb{S}^{n-1}$ implies $\|\nu\|_{1} \geq 1$, with equality only if $\nu$ coincides with one of the vectors of the orthonormal basis $\left(e_{1}, \ldots, e_{n}\right)$ of $\mathbb{R}^{n}$. In particular

$$
\mathcal{E}(u, \Omega) \leq \mathcal{E}_{0}(u, \Omega) .
$$

From our assumptions on $\theta$, we have that $\mathcal{E}(u, \Omega)$ decreases under truncation. By the lower semicontinuity of $\mathcal{E}(u, \Omega)$, this implies that setting $u_{T}:=\max (-T, \min (T, u))$ for any $T>0$, then $\mathcal{E}\left(u_{T}, \Omega\right)$ converges to $\mathcal{E}(u, \Omega)$ as $T \uparrow+\infty$. Hence, with no loss of generality, we can assume that $u \in L^{\infty}(\Omega)$.

We divide the proof into five steps. In the first step we construct a suitable discrete approximation $u_{\varepsilon}^{y}$ of $u$, defined on points of a lattice. For any $\delta>0$ set

$$
\Omega^{\delta}:=\left\{x \in \mathbb{R}^{n}: \operatorname{dist}(x, \Omega)<\delta\right\} .
$$

Step 1. Let $\varepsilon>0$ be such that $\Omega_{\varepsilon}:=\Omega^{2 n^{1 / 2} \varepsilon} \subset \subset \Omega^{\prime}$ and, for any $y \in[0,1)^{n}$, set

$$
u_{\varepsilon}^{y}(x):=u(x+\varepsilon y), \quad x \in \varepsilon \mathbb{Z}^{n} \cap \Omega^{\prime} .
$$

Define the discrete energy $D_{\varepsilon}^{y}$ of $u_{\varepsilon}^{y}$ as

$$
D_{\varepsilon}^{y}:=\varepsilon^{n-1} \sum_{i=1}^{n} \sum_{x, x+\varepsilon e_{i} \in \Omega_{\varepsilon / 2}} \theta\left(u_{\varepsilon}^{y}\left(x+\varepsilon e_{i}\right)-u_{\varepsilon}^{y}(x)\right) .
$$

where $\Omega_{\varepsilon / 2}:=\Omega^{n^{1 / 2} \varepsilon}$. We claim that

$$
\int_{(0,1)^{n}} D_{\varepsilon}^{y} d y \leq \mathcal{E}_{0}\left(u, \Omega_{\varepsilon}\right)
$$

To show the claim, we will follow some arguments in [34], [17] [18] and [19]. Let us first establish an inequality in one dimension: given a bounded open interval $I \subset \mathbb{R}, \varepsilon>0$ sufficiently small and $v \in \mathcal{P}(I)$, we have

$$
\varepsilon^{-1} \int_{I \cap(I-\varepsilon)} \theta(v(t+\varepsilon)-v(t)) d t \leq \sum_{t \in J_{v}} \theta\left(v^{+}(t)-v^{-}(t)\right) .
$$

Indeed, for almost any $t \in I \cap(I-\varepsilon)$, using the subadditivity of $\theta$ it follows

$$
\theta(v(t+\varepsilon)-v(t)) \leq \sum_{s \in J_{v} \cap(t, t+\varepsilon)} \theta\left(v^{+}(s)-v^{-}(s)\right) .
$$

Therefore, using Fubini's Theorem,

$$
\begin{aligned}
\int_{I \cap(I-\varepsilon)} \theta(v(t+\varepsilon)-v(t)) d t & \leq \int_{I \cap(I-\varepsilon)} \sum_{s \in J_{v}} 1_{(t, t+\varepsilon)}(s) \theta\left(v^{+}(s)-v^{-}(s)\right) d t \\
& =\sum_{s \in J_{v}} \theta\left(v^{+}(s)-v^{-}(s)\right) \int_{I \cap(I-\varepsilon)} 1_{(t, t+\varepsilon)}(s) d t
\end{aligned}
$$


which implies (4.5).

We can now prove claim (4.4). Since

$$
\left.\int_{(0,1)^{n}} D_{\varepsilon}^{y} d y=\varepsilon^{n-1} \sum_{i=1}^{n} \sum_{x, x+\varepsilon e_{i} \in \Omega_{\varepsilon / 2}} \int_{(0,1)^{n}} \theta\left(u\left(x+\varepsilon y+\varepsilon e_{i}\right)\right)-u(x+\varepsilon y)\right) d y,
$$

making the variable change $x^{\prime}:=\varepsilon y+x($ for fixed $x$ ) gives

$$
\begin{aligned}
\int_{(0,1)^{n}} D_{\varepsilon}^{y} d y & \leq \varepsilon^{-1} \sum_{i=1}^{n} \int_{\Omega_{\varepsilon / 2}} \theta\left(u\left(x^{\prime}+\varepsilon e_{i}\right)-u\left(x^{\prime}\right)\right) d x^{\prime} \\
& =\varepsilon^{-1} \sum_{i=1}^{n} \int_{\Pi_{e_{i}}} \int_{\left(\Omega_{\varepsilon / 2}\right)_{e_{i} \zeta}} \theta\left(u\left(\zeta+(t+\varepsilon) e_{i}\right)-u\left(\zeta+t e_{i}\right)\right) d t d \mathcal{H}^{n-1}(\zeta) .
\end{aligned}
$$

If, for any $i=1, \ldots, n$ and $\mathcal{H}^{n-1}$-almost every $\zeta \in \Pi_{e_{i}}$ we define as in Section 2.2, the onedimensional slice $u_{e_{i} \zeta}$ as $u_{e_{i} \zeta}(t):=u\left(\zeta+t e_{i}\right)$ for almost each $t \in \mathbb{R}$ such that $\zeta+t e_{i} \in \Omega^{\prime}$, we get,

$$
\int_{(0,1)^{n}} D_{\varepsilon}^{y} d y \leq \varepsilon^{-1} \sum_{i=1}^{n} \int_{\Pi_{e_{i}}} \int_{\left(\Omega_{\varepsilon / 2}\right)_{e_{i} \zeta}} \theta\left(u_{e_{i} \zeta}(t+\varepsilon)-u_{e_{i} \zeta}(t)\right) d t d \mathcal{H}^{n-1}(\zeta) .
$$

Hence, using (4.5),

$$
\int_{(0,1)^{n}} D_{\varepsilon}^{y} d y \leq \sum_{i=1}^{n} \int_{\Pi_{e_{i}}} \sum_{s \in J_{u_{i} \zeta} \cap\left(\Omega_{\varepsilon}\right)_{e_{i} \zeta}} \theta\left(u_{e_{i} \zeta}^{+}(s)-u_{e_{i} \zeta}^{-}(s)\right) d \mathcal{H}^{n-1}(\zeta) .
$$

Therefore, by (2.5),

$$
\int_{(0,1)^{n}} D_{\varepsilon}^{y} d y \leq \sum_{i=1}^{n} \int_{J_{u} \cap \Omega_{\varepsilon}}\left|\left\langle\nu_{u}, e_{i}\right\rangle\right| \theta\left(u^{+}-u^{-}\right) d \mathcal{H}^{n-1}=\mathcal{E}_{0}\left(u, \Omega_{\varepsilon}\right),
$$

which is claim (4.4). The proof of Step 1 is concluded.

In the next step we define a suitable piecewise constant interpolation $\bar{u}_{\varepsilon}^{y}$ of $u_{\varepsilon}^{y}$ having the property that $\mathcal{E}\left(\bar{u}_{\varepsilon}^{y}, \Omega\right)$ and $\mathcal{E}_{0}\left(\bar{u}_{\varepsilon}^{y}, \Omega\right)$ coincide.

Step 2. Let $\varepsilon$ and $y$ be as in Step 1. Define the function $\bar{u}_{\varepsilon}^{y}: \Omega \rightarrow \mathbb{R}$ as

$$
\bar{u}_{\varepsilon}^{y}(z):=\sum_{x \in \varepsilon \mathbb{Z}^{n}} u_{\varepsilon}^{y}(x) 1_{C_{\varepsilon}^{y}(x)}(z), \quad z \in \Omega
$$

where $C_{\varepsilon}^{y}(x)$ denotes the open coordinate hypercube centered at $x+\varepsilon y$ of side $\varepsilon$, i.e.,

$$
C_{\varepsilon}^{y}(x):=x+\varepsilon y+(-\varepsilon / 2, \varepsilon / 2)^{n} .
$$

Clearly $\bar{u}_{y}^{\varepsilon} \in \mathcal{P}(\Omega)$ and its jump set is contained in the union of the facets of the hypercubes $C_{\varepsilon}^{y}(x)$. Let us prove that

(i) $\lim _{\varepsilon \rightarrow 0} \int_{(0,1)^{n}}\left\|\bar{u}_{\varepsilon}^{y}-u\right\|_{L^{1}(\Omega)} d y=0$; 
(ii) $\mathcal{E}\left(\bar{u}_{\varepsilon}^{y}, \Omega\right)=\mathcal{E}_{0}\left(\bar{u}_{\varepsilon}^{y}, \Omega\right) \leq D_{\varepsilon}^{y}$.

To show (i) and (ii), we assume without loss of generality that all functions are extended to 0 outside $\Omega^{\prime}$. Making the variables change

$$
(x, y) \in \varepsilon \mathbb{Z}^{n} \times[0,1)^{n} \mapsto y^{\prime}:=\varepsilon y+x
$$

and then

$$
y^{\prime} \in \mathbb{R}^{n} \rightarrow \xi=z-y^{\prime} \quad(\text { for fixed } z),
$$

we obtain

$$
\begin{aligned}
\int_{(0,1)^{n}}\left\|\bar{u}_{\varepsilon}^{y}-u\right\|_{L^{1}(\Omega)} d y & =\int_{(0,1)^{n}} \int_{\mathbb{R}^{n}}\left|\sum_{x \in \varepsilon \mathbb{Z}^{n}} u(\varepsilon y+x) 1_{C_{\varepsilon}^{y}(x)}(z)-u(z)\right| d z d y \\
& =\int_{(0,1)^{n}} \int_{\mathbb{R}^{n}}\left|\sum_{x \in \varepsilon \mathbb{Z}^{n}}(u(\varepsilon y+x)-u(z)) 1_{C_{\varepsilon}^{y}(x)}(z)\right| d z d y \\
& \leq \int_{(0,1)^{n}} \sum_{x \in \varepsilon \mathbb{Z}^{n}} \int_{\mathbb{R}^{n}}|u(\varepsilon y+x)-u(z)| 1_{C_{\varepsilon}^{y}(x)}(z) d z d y \\
& =\varepsilon^{-n} \int_{\mathbb{R}^{n}} \int_{\mathbb{R}^{n}}\left|u\left(y^{\prime}\right)-u(z)\right| 1_{\left(-\frac{\varepsilon}{2}, \frac{\varepsilon}{2}\right)^{n}}\left(z-y^{\prime}\right) d z d y^{\prime} \\
& =\varepsilon^{-n} \int_{\mathbb{R}^{n}} \int_{\mathbb{R}^{n}}|u(z-\xi)-u(z)| 1_{\left(-\frac{\varepsilon}{2}, \frac{\varepsilon}{2}\right)^{n}}(\xi) d z d \xi \\
& =\varepsilon^{-n} \int_{\left(-\frac{\varepsilon}{2}, \frac{\varepsilon}{2}\right)^{n}}\|u(\cdot-\xi)-u(\cdot)\|_{L^{1}\left(\mathbb{R}^{n}\right)} d \xi \\
& =\int_{\left(-\frac{1}{2}, \frac{1}{2}\right)^{n}}\|u(\cdot-\varepsilon \xi)-u(\cdot)\|_{L^{1}\left(\mathbb{R}^{n}\right)} d \xi
\end{aligned}
$$

which tends to zero as $\varepsilon \rightarrow 0$ by the dominated convergence theorem. This proves (i). The equality in (ii) follows from the fact that

$$
\left\|\nu_{\bar{u}_{\varepsilon}^{y}}(x)\right\|_{1}=1 \quad \text { for } \mathcal{H}^{n-1} \text { - a.e. } x \in J_{\bar{u}_{\varepsilon}^{y}},
$$

since the jump of $\bar{u}_{\varepsilon}^{y}$ is contained in facets of coordinate hypercubes.

The inequality in (ii) follows from the definition of $\mathcal{E}_{0}$ (see (4.2)) and definitions (4.3) of $D_{\varepsilon}^{y}$ and (4.6) of $\bar{u}_{\varepsilon}^{y}$.

From (i) we deduce that there exists a set $A \subset(0,1)^{n}$ with $|A|=1$ and a subsequence $\left(\varepsilon_{k}\right) \subset(0,1)$ converging to zero such that $\bar{u}_{\varepsilon_{k}}^{y} \rightarrow u$ in $L^{1}(\Omega)$ as $k \rightarrow \infty$ for any $y \in A$.

From Step 1 and Fatou's lemma we have

$$
\int_{(0,1)^{n}} \liminf _{k \rightarrow+\infty} D_{\varepsilon_{k}}^{y} d y \leq \mathcal{E}_{0}(u, \Omega) .
$$

Hence there exists a point $\bar{y} \in A$ and a further subsequence (still denoted by $\left(\varepsilon_{k}\right)$ ) such that in addition there exists the $\lim _{k \rightarrow+\infty} D_{\varepsilon_{k}}^{\bar{y}}$ and

$$
\lim _{k \rightarrow+\infty} D_{\varepsilon_{k}}^{\bar{y}} \leq \mathcal{E}_{0}(u, \Omega) .
$$


Summarizing, we have shown that given $u \in \mathcal{P}(\Omega) \cap L^{\infty}(\Omega)$, if we set

$$
u_{k}:=u_{\varepsilon_{k}}^{\bar{y}}, \quad k \in \mathbb{N},
$$

the sequence $\left(u_{k}\right) \subset \mathcal{P}(\Omega)$ has the following properties:

(a) $\left\|u_{k}\right\|_{L^{\infty}(\Omega)} \leq\|u\|_{L^{\infty}(\Omega)}$ for any $k \in \mathbb{N}$,

(b) $\lim _{k \rightarrow \infty}\left\|u_{k}-u\right\|_{L^{1}(\Omega)}=0$,

(c) $\limsup _{k \rightarrow \infty} \mathcal{E}_{0}\left(u_{k}, \Omega\right) \leq \mathcal{E}_{0}(u, \Omega)$,

(d) $\mathcal{E}_{0}\left(u_{k}, \Omega\right)=\mathcal{E}\left(u_{k}, \Omega\right)$ and $J_{u_{k}}$ is contained in a finite union of facets of coordinate hypercubes for any $k$.

To prove the theorem, it remains to show that we can replace $\mathcal{E}_{0}(u, \Omega)$ with $\mathcal{E}(u, \Omega)$ on the right hand side of the inequality in assertion (c).

Step 3. Let $A \subset \mathbb{R}^{n}$ be a bounded open set with Lipschitz boundary and let $v \in \mathcal{P}(A) \cap L^{\infty}(A)$ be with $\mathcal{E}(v, A)<+\infty$. Let $\left(v_{k}\right) \subset \mathcal{P}(A)$ be a sequence converging to $v$ in $L^{1}(A)$ such that $\left\|v_{k}\right\|_{L^{\infty}(A)} \leq\|v\|_{L^{\infty}(A)}$ for any $k \in \mathbb{N}$ and $\lim _{k \rightarrow \infty} \mathcal{E}_{0}\left(v_{k}, A\right)=\mathcal{E}_{0}(v, A)$. Then

$$
\lim _{k \rightarrow \infty} \int_{\partial A}\left|T v_{k}-T v\right| d \mathcal{H}^{n-1}=0,
$$

where $T v_{k}$ (resp. $T v$ ) denotes the trace of $v_{k}$ (resp. of $v$ ) on $\partial A$.

This follows from the fact that, if $v \in \mathcal{P}(A) \cap L^{\infty}(A)$, then there exists a constant $c>$ 0 depending only $\theta$ and $\|v\|_{L^{\infty}(A)}$ such that $\int_{B}|D v| \leq c \mathcal{E}_{0}(v, B)$ for any open set $B \subseteq$ $A$. A careful analysis of [6, Theorem 3.88] (see also [29, Section 5.3]), the $L^{1}(A)$-lower semicontinuity of $v \rightarrow \mathcal{E}_{0}(v, B)$ for any open set $B \subseteq A$ and the fact that $\mathcal{E}_{0}(v, \cdot)$ is a regular measure, imply (4.7).

Step 4 . Let $\mu$ be a Radon measure on $\Omega$ with values in $\mathbb{R}^{d}, d \geq 1$, with total variation measure $|\mu|$ and $|\mu|(\Omega)<+\infty$. For any $h \in \mathbb{N}$, let us consider a family $\left\{B_{k}^{h}\right\}$, where $B_{k}^{h}$ are nonempty Borel subsets of $\Omega$ with $B_{k_{1}}^{h} \cap B_{k_{2}}^{h}=\emptyset$ when $k_{1}, k_{2} \in \mathbb{N}$ and $k_{1} \neq k_{2}$, and

$$
|\mu|\left(\Omega \backslash \bigcup_{k} B_{k}^{h}\right)=0
$$

If in addition

$$
\lim _{h \rightarrow+\infty} \sup _{k \in \mathbb{N}} \operatorname{diam}\left(B_{k}^{h}\right)=0,
$$

then

$$
|\mu|(\Omega)=\lim _{h \rightarrow+\infty} \sum_{k}\left|\mu\left(B_{k}^{h}\right)\right| .
$$

To prove (4.10) it is enough to show that for any $\varphi \in\left(\mathcal{C}_{c}(\Omega)\right)^{d}$ with $\|\varphi\|_{L^{\infty}(\Omega)} \leq 1$, we have

$$
\int_{\Omega}\langle\varphi, \mu\rangle \leq \lim _{h \rightarrow+\infty} \sum_{k}\left|\mu\left(B_{k}^{h}\right)\right|
$$


For any $h, k \in \mathbb{N}$ pick a point $x_{k}^{h} \in B_{k}^{h}$, and define

$$
\varphi_{h}(x):=\sum_{k} \varphi\left(x_{k}^{h}\right) 1_{B_{k}^{h}}(x), \quad x \in \Omega .
$$

Using the uniform continuity of $\varphi$ and assumption (4.9), we have $\lim _{h \rightarrow+\infty}\left\|\varphi-\varphi_{h}\right\|_{L^{\infty}(\Omega, \mu)}=$ 0 . Therefore

$$
\int_{\Omega}\langle\varphi, \mu\rangle=\int_{\Omega}\left\langle\varphi_{h}, \mu\right\rangle+\int_{\Omega}\left\langle\left(\varphi-\varphi_{h}\right), \mu\right\rangle \leq \sum_{k}\left|\mu\left(B_{k}^{h}\right)\right|+o(1),
$$

and (4.10) follows.

In the next (final) step we show that it is possible to replace $\mathcal{E}_{0}(u, \Omega)$ with $\mathcal{E}(u, \Omega)$ in (c), by suitably modifying the sequence $\left(u_{k}\right)$.

Step 5. Let $\delta>0$ and $y \in(0, \delta)^{n}$. For each $z \in \delta \mathbb{Z}^{n}$ let us denote by $C_{\delta}(z)$ the open hypercube $y+z+(0, \delta)^{n}$. Possibly modifying the choice of the origin $y$, we can assume that

$$
|D u|\left(\Omega \backslash \bigcup_{z \in \delta \mathbb{Z}^{n}} C_{\delta}(z)\right)=0
$$

We will apply the construction described in Steps 1,2 to each of the sets $\Omega \cap C_{\delta}(z)$ and then glue together the sequences thus obtained. Notice that it may happen that the set $\Omega \cap C_{\delta}(z)$ is not Lipschitz (if non empty); however we already know that $u$ is defined on $\Omega^{\prime}$ which strictly contains $\Omega$. Let us define the vector-valued Radon measure $\mu$ as

$$
\mu(B):=\int_{B \cap J_{u}} \theta\left(u^{+}-u^{-}\right) \nu_{u} d \mathcal{H}^{n-1}, \quad B \text { Borel set } \subseteq \Omega .
$$

Observe that $|\mu|(B)=\int_{B \cap J_{u}} \theta\left(u^{+}-u^{-}\right) d \mathcal{H}^{n-1}$.

With any hypercube $C_{\delta}(z)$ we associate a unit vector $\xi_{z} \in S^{n-1}$ such that

$$
\left\langle\xi_{z}, \mu\left(\Omega \cap C_{\delta}(z)\right)\right\rangle=\left|\mu\left(\Omega \cap C_{\delta}(z)\right)\right| .
$$

If we consider an orthonormal basis of $\mathbb{R}^{n}$ having $\xi_{z}$ as the first vector and if we let $\|\cdot\|_{1, z}$ be the $\|\cdot\|_{1}$ norm in this basis, from Steps 1 and 2 we can find a sequence $\left(u_{k}^{\delta}\right) \subset L^{1}\left(\Omega \cap C_{\delta}(z)\right)$ converging to $u$ in $L^{1}\left(\Omega \cap C_{\delta}(z)\right)$ and satisfying properties (a)-(d) with $\Omega \cap C_{\delta}(z)$ in place of $\Omega$, and such that

$$
\begin{aligned}
\limsup _{k \rightarrow \infty} \mathcal{E}\left(u_{k}^{\delta}, \Omega \cap C_{\delta}(z)\right) & \leq \int_{J_{u} \cap C_{\delta}(z)}\left\|\nu_{u}\right\|_{1, z} d|\mu| \\
& \leq \int_{J_{u} \cap C_{\delta}(z)}\left|\left\langle\xi_{z}, \nu_{u}\right\rangle\right| d|\mu|+c \int_{J_{u} \cap C_{\delta}(z)} \sqrt{1-\left\langle\xi_{z}, \nu_{u}\right\rangle^{2}} d|\mu| \\
& =\mathcal{E}\left(u, \Omega \cap C_{\delta}(z)\right)+c \int_{J_{u} \cap C_{\delta}(z)} \sqrt{1-\left\langle\xi_{z}, \nu_{u}\right\rangle^{2}} d|\mu|,
\end{aligned}
$$


where $c>0$ is a constant controlling the euclidean norm of a vector with its $\|\cdot\|_{1}$-norm. Using the same construction for all $z$ such that $J_{u} \cap C_{\delta}(z) \neq \emptyset$, and gluing together the functions obtained in each hypercube, we construct $u_{k} \in L^{1}(\Omega)$ such that

$$
\mathcal{E}\left(u_{k}, \Omega\right)=\sum_{z \in \delta \mathbb{Z}^{n}} \mathcal{E}\left(u_{k}, \Omega \cap C_{\delta}(z)\right)+\int_{\Omega \backslash} \bigcup_{z \in \delta \mathbb{Z}^{n}} C_{\delta}(z) \theta\left(u_{k}^{+}-u_{k}^{-}\right) d \mathcal{H}^{n-1} .
$$

We notice that the jump set of the $u_{k}$ 's consists of the union of the facets of the lattice and of the jump set inside each hypercubes. Recalling (4.12) and Step 3, possibly passing to a (not relabelled) subsequence, we can assume that the traces of $u_{k}$ on both sides of each facet of a hypercube $C_{\delta}(z)$ converge $|\mu|$-almost everywhere to the same limit (which is the trace of $u$ ) as $k \rightarrow+\infty$, hence the last term on the right hand side of (4.14) tends to zero by the continuity of $\theta$ at 0 . Therefore there exists $\bar{k} \in \mathbb{N}$ such that, if $k>\bar{k}$, then $\left\|u_{k}-u\right\|_{L^{1}(\Omega)}<\delta$ and

$$
\begin{aligned}
\mathcal{E}\left(u_{k}, \Omega\right) & \leq \sum_{z \in \delta \mathbb{Z}^{n}}\left[\int_{J_{u} \cap C_{\delta}(z)}\left|\left\langle\xi_{z}, \nu_{u}\right\rangle\right| d|\mu|+c \int_{J_{u} \cap C_{\delta}(z)} \sqrt{1-\left\langle\xi_{z}, \nu_{u}\right\rangle^{2}} d|\mu|\right]+\delta \\
& \leq \mathcal{E}(u, \Omega)+c \sum_{z \in \delta \mathbb{Z}^{n}} \int_{J_{u} \cap C_{\delta}(z)} \sqrt{1-\left\langle\xi_{z}, \nu_{u}\right\rangle^{2}} d|\mu|+\delta
\end{aligned}
$$

where in the last inequality we made use of (4.13). The proof is therefore achieved if we show that the last sum on the right hand side of (4.15) can be made small for $\delta>0$ sufficiently small. We have

$$
\begin{aligned}
& \sum_{z \in \delta \mathbb{Z}^{n}} \int_{J_{u} \cap C_{\delta}(z)} \sqrt{1-\left\langle\xi_{z}, \nu_{u}\right\rangle^{2}} d|\mu| \\
= & \sum_{z \in \delta \mathbb{Z}^{n}} \int_{J_{u} \cap C_{\delta}(z)} \sqrt{\left(1-\left\langle\nu_{u}, \xi_{z}\right\rangle\right)\left(1+\left\langle\nu_{u}, \xi_{z}\right\rangle\right)} d|\mu| \\
\leq & \left(\sum_{z \in \delta \mathbb{Z}^{n}} \int_{J_{u} \cap C_{\delta}(z)}\left(1-\left\langle\nu_{u}, \xi_{z}\right\rangle\right) d|\mu|\right)^{1 / 2} \sqrt{2|\mu|(\Omega)} .
\end{aligned}
$$

Observing that from (4.13)

$$
\int_{J_{u} \cap C_{\delta}(z)}\left(1-\left\langle\nu_{u}, \xi_{z}\right\rangle\right) d|\mu| \leq|\mu|(\Omega)-\sum_{z \in \delta \mathbb{Z}^{n}}\left|\mu\left(\Omega \cap C_{\delta}(z)\right)\right|,
$$

by Step 4 we see that the right hand side of (4.16) can be made arbitrarily small if $\delta>0$ is small enough. This concludes the proof.

\section{Equicoerciveness and $\Gamma$-convergence}

Using a slicing argument and Theorem 4.1, the results of Section 3 can be generalized in $n$-dimensions. 
We remind that $F_{\nu}: L^{2}(\Omega) \rightarrow[0,+\infty]$ and $\mathcal{F}: L^{2}(\Omega) \rightarrow[0,+\infty]$ are defined in (1.1) and (1.3), respectively. If $A \in \mathcal{A}(\Omega)$ and $u \in H^{2}(A)$ we define the localized functional $F_{\nu}(u, A)$ as

$$
F_{\nu}(u, A):=\frac{1}{2} \int_{A}\left[\nu^{3}\left|\nabla^{2} u\right|^{2}+\frac{1}{\nu \phi(1 / \nu)} \phi(|\nabla u|)\right] d x .
$$

Similarly, if $u \in X(A) \cap L^{2}(A)$, we set

$$
\mathcal{F}(u, A):=\sigma_{a} \int_{A \cap J_{u}}\left|u^{+}-u^{-}\right|^{\frac{2+a}{4-a}} d \mathcal{H}^{n-1}
$$

We will need the following lemma on the supremum of a family of measures (see [15]).

Lemma 5.1. Let $\mu: \mathcal{A}(\Omega) \rightarrow[0,+\infty)$ be a superadditive set function and let $\lambda$ be a positive measure on $\Omega$. For any $i \in \mathbb{N}$ let $\psi_{i}$ be a Borel function on $\Omega$ such that $\mu(A) \geq \int_{A} \psi_{i} d \lambda$ for all $A \in \mathcal{A}(\Omega)$. Then

$$
\mu(A) \geq \int_{A} \psi d \lambda \quad \forall A \in \mathcal{A}(\Omega)
$$

where $\psi:=\sup _{i \in \mathbb{N}} \psi_{i}$.

We start our $n$-dimensional analysis with the following two results, which are independent of Lemma 4.1.

Theorem 5.2 (Equicoerciveness). Let $\left(u_{\nu}\right) \subset H^{2}(\Omega)$ be a sequence satisfying

$$
\sup _{\nu} F_{\nu}\left(u_{\nu}, \Omega\right)<+\infty
$$

and such that $\int_{\Omega} u_{\nu} d x=0$. Then there exist a function $u \in X(\Omega) \cap L^{2}(\Omega)$ and a subsequence of $\left(u_{\nu}\right)$ weakly* converging to $u$ in $B V(\Omega)$.

Proof. By Lemma 3.1, there exist $\bar{\nu} \in(0,1]$, a decreasing function $\omega:(0, \bar{\nu}) \rightarrow(0,+\infty)$ with $\lim _{\nu \rightarrow 0^{+}} \omega(s)=0$ and a constant $C>0$ such that for every interval $I \subset \mathbb{R}$, every $v \in H^{2}(I)$ and $\nu \in(0, \bar{\nu})$, inequality (3.1) holds, namely, for $\nu \in(0, \bar{\nu})$,

$$
\int_{I}\left|v^{\prime}\right| d x \leq|I| \omega(\nu)+C F_{\nu}(v, I) .
$$

Therefore, if $v \in H^{2}(\Omega)$ and $\xi \in \mathbb{S}^{n-1}$, by (5.5),

$$
\begin{aligned}
\int_{\Omega}|\langle\nabla v, \xi\rangle| d x & =\int_{\Pi_{\xi}} \int_{\Omega_{\xi y}}|\langle\nabla v(y+t \xi), \xi\rangle| d t d \mathcal{H}^{n-1}(y) \\
& =\int_{\Pi_{\xi}} \int_{\Omega_{\xi y}}\left|v_{\xi y}^{\prime}\right| d t d \mathcal{H}^{n-1}(y) \\
& \leq \int_{\Pi_{\xi}}\left(\left|\Omega_{\xi y}\right| \omega(\nu)+C F_{\nu}\left(v_{\xi y}, \Omega_{\xi y}\right)\right) d \mathcal{H}^{n-1}(y) \\
& \leq|\Omega| \omega(\nu)+C F_{\nu}(v, \Omega) .
\end{aligned}
$$


Letting $\xi=e_{i}$ and summing (5.2) over $i \in\{1, \ldots, n\}$, we deduce using (5.1), that there exists a positive constant $\kappa$ such that

$$
\sup _{\nu} \int_{\Omega}\left|\nabla v_{\nu}\right| d x \leq \kappa \sup _{\nu}\left(|\Omega| \omega(\nu)+F_{\nu}\left(v_{\nu}, \Omega\right)\right)<+\infty .
$$

Thus if $\int_{\Omega} u_{\nu} d x=0$ then there exist a function $u \in B V(\Omega)$ and a subsequence of $\left(u_{\nu}\right)$ weakly* converging to $u$ in $B V(\Omega)$.

Theorem 5.3 (Г-liminf). We have

$$
\mathcal{F}(\cdot, A) \leq \Gamma\left(L^{2}(\Omega)\right)-\lim _{\nu \rightarrow 0^{+}} F_{\nu}(\cdot, A), \quad A \in \mathcal{A}(\Omega) .
$$

Proof. The proof follows closely [4, Prop. 3.4]. Fix $\xi \in \mathbb{S}^{n-1}, u \in H^{2}(\Omega), A \in \mathcal{A}(\Omega)$ and let as usual $u_{\xi y}: A_{\xi y} \rightarrow \mathbb{R}$ be the slice defined as in Section 2.2, i.e.,

$$
u_{\xi y}(t):=u(y+t \xi), \quad t \in A_{\xi y} .
$$

Since for almost every $t \in A_{\xi y}$ and $\mathcal{H}^{n-1}$-almost every $y$

$$
u_{\xi y}^{\prime}(t)=\langle\nabla u(y+t \xi), \xi\rangle \quad \text { and } \quad u_{\xi y}^{\prime \prime}=\left\langle\nabla^{2} u(y+t \xi) \xi, \xi\right\rangle,
$$

by Fubini's Theorem and being $\phi$ nondecreasing,

$$
\begin{aligned}
& F_{\nu}(u, A) \\
= & \int_{\Pi_{\xi}}\left[\frac{1}{2} \int_{A_{\xi y}} \nu^{3}\left|\nabla^{2} u(y+t \xi)\right|^{2}+\frac{1}{\nu \phi(1 / \nu)} \phi(|\nabla u(y+t \xi)|) d t\right] d \mathcal{H}^{n-1}(y) \\
\geq & \int_{\Pi_{\xi}}\left[\frac{1}{2} \int_{A_{\xi y}} \nu^{3}\left(\left\langle\nabla^{2} u(y+t \xi) \xi, \xi\right\rangle\right)^{2}+\frac{1}{\nu \phi(1 / \nu)} \phi(\langle\nabla u(y+t \xi) \xi, \xi\rangle) d t\right] d \mathcal{H}^{n-1}(y) \\
= & \int_{\Pi_{\xi}} F_{\nu}\left(u_{\xi y}, A_{\xi y}\right) d \mathcal{H}^{n-1}(y) .
\end{aligned}
$$

Let now $\left(u_{\nu}\right) \subset H^{2}(A)$ be a sequence converging to $u$ in $L^{2}(A)$ as $\nu \rightarrow 0^{+}$. By Fubini's theorem and Fatou's lemma we have $\left(u_{\nu}\right)_{\xi y} \rightarrow u_{\xi y}$ in $L^{2}\left(A_{\xi y}\right)$ for $\mathcal{H}^{n-1}$-almost every $y \in \Pi_{\xi}$. Hence by (5.5) and Theorem 3.3,

$$
\sigma_{a} \sum_{x \in J_{u_{\xi y}} \cap A_{\xi y}}\left|u_{\xi y}\left(x^{+}\right)-u_{\xi y}\left(x^{-}\right)\right|^{\frac{2+a}{4-a}} \leq \liminf _{\nu \rightarrow 0^{+}} F_{\nu}\left(\left(u_{\nu}\right)_{\xi y}, A_{\xi y}\right) .
$$

Thus, applying once more Fatou's lemma,

$$
\int_{\Pi_{\xi}}\left(\sigma_{a} \sum_{x \in J_{u_{\xi y}} \cap A_{\xi y}}\left|u_{\xi y}\left(x^{+}\right)-u_{\xi y}\left(x^{-}\right)\right|^{\frac{2+a}{4-a}}\right) d \mathcal{H}^{n-1}(y) \leq \liminf _{\nu \rightarrow 0^{+}} F_{\nu}\left(u_{\nu}, A\right) .
$$


Let us first show that

$$
u \in X(\Omega) .
$$

Let $T>0$ and set $u_{T}:=\max (-T, \min (T, u))$. By the results of Section 3 we know that $\left(u_{T}\right)_{\xi y} \in X\left(A_{\xi y}\right)$ and thus by (5.6) and (5.1),

$$
\int_{\Pi_{\xi}}\left|D\left(u_{T}\right)_{\xi y}\right|\left(A_{\xi y}\right) d \mathcal{H}^{n-1}(y)<+\infty, \quad \xi \in \mathbb{S}^{n-1}, \mathcal{H}^{n-1}-\text { a.e. } y \in \Pi_{\xi} .
$$

Therefore, by Theorem 2.2 it follows that $u \in G S B V(A)$. By (5.3) we also know that $u \in B V(A)$ and thus $u \in S B V(A)$. Now for every $\xi \in \mathbb{S}^{n-1}$,

$$
\begin{aligned}
\int_{A}|\langle\nabla u, \xi\rangle| d x & =\int_{\Pi_{\xi}} \int_{A_{\xi y}}|\langle\nabla u(y+t \xi), \xi\rangle| d t d \mathcal{H}^{n-1}(y) \\
& =\int_{\Pi_{\xi}} \int_{A_{\xi y}}\left|u_{\xi y}^{\prime}(t)\right| d t d \mathcal{H}^{n-1}(y) \\
& =0 .
\end{aligned}
$$

Hence $\nabla u=0$ almost everywhere, so that $u \in X(A)$. This concludes the proof of (5.7).

By Theorem 2.2 (a), we get

$$
\liminf _{\nu \rightarrow 0^{+}} F_{\nu}\left(u_{\nu}, A\right) \geq \sigma_{a} \int_{A \cap J_{u}}\left|u\left(x^{+}\right)-u\left(x^{-}\right)\right|^{\frac{2+a}{4-a}}\left|\left\langle\nu_{u}, \xi\right\rangle\right| d \mathcal{H}^{n-1}(y) .
$$

We now let $\gamma$ be the superadditive increasing set function defined on $\mathcal{A}(\Omega)$ by

$$
\gamma(A):=\Gamma-\liminf _{\nu \rightarrow 0^{+}} F_{\nu}(u, A)
$$

and we let $\lambda$ be the Radon measure defined as

$$
\lambda:=\left|u\left(x^{+}\right)-u\left(x^{-}\right)\right|^{\frac{2+a}{4-a}} \mathcal{H}^{n-1}\left\llcorner J_{u} .\right.
$$

Fix a sequence $\left(\xi_{i}\right)_{i \in \mathbb{N}}$ dense in $\mathbb{S}^{n-1}$. By (5.8) we have

$$
\gamma(A) \geq \int_{A} \psi_{i} d \lambda, \quad i \in \mathbb{N},
$$

where

$$
\psi_{i}(x):= \begin{cases}\left|\left\langle\nu_{u}(x), \xi_{i}\right\rangle\right| & \text { if } x \in J_{u} \\ 0 & \text { if } x \in A \backslash J_{u} .\end{cases}
$$

Hence by Lemma 5.1,

$$
\Gamma-\liminf _{\nu \rightarrow 0^{+}} F_{\nu}(u, A) \geq \int_{A} \sup _{i} \psi_{i}(x) d \lambda=\mathcal{F}(u, A) .
$$

We next pass to the proof of the $\Gamma$-limsup inequality. We start by studying the particular case $u=s 1_{E}$, adapting the proof from [4, Prop 3.5]. 
Proposition 5.4. Let $\Omega^{\prime} \supset \supset \Omega$ be a bounded open set and let $E$ be such that $E=E^{\prime} \cap \Omega$ where $E^{\prime}$ is a set of finite perimeter in $\Omega^{\prime}$ such that $\partial E^{\prime} \cap \Omega^{\prime}$ is a smooth hypersurface. Then

$$
\Gamma-\limsup _{\nu \rightarrow 0^{+}} F_{\nu}\left(s 1_{E}, A\right) \leq \sigma_{a}|s|^{\frac{2+a}{4-a}} \mathcal{H}^{n-1}(A \cap \partial E),
$$

for all $s \in \mathbb{R}$ and $A \in \mathcal{A}(\Omega)$.

Proof. Let

$$
d(x):=\operatorname{dist}\left(x, \mathbb{R}^{n} \backslash E^{\prime}\right)-\operatorname{dist}\left(x, E^{\prime}\right), \quad x \in \mathbb{R}^{n},
$$

be the signed distance function from $\partial E^{\prime}$ positive in $E^{\prime}$. Take $\delta>0$ such that $d \in \mathcal{C}^{\infty}\left(\overline{V_{\delta} \cap \Omega}\right)$ where $V_{\delta}:=\left\{x \in \mathbb{R}^{n}:|d(x)|<\delta\right\}$ (see for instance [5]), and recall that $|\nabla d|^{2}=1$ in $V_{\delta} \cap \Omega$. Let $b>0$ and $\left(u_{\nu}\right)$ be the one-dimensional recovery sequence given in Section 3 converging to $s 1_{\{t>0\}}$ in $L^{2}((-\delta, \delta))$, so that

$$
\lim _{\nu \rightarrow 0^{+}} F_{\nu}\left(u_{\nu},(-\delta, \delta)\right)=\sigma_{a}|s|^{\frac{2+a}{4-a}}+b .
$$

We next define

$$
\widetilde{u}_{\nu}(x):=u_{\nu}(d(x)), \quad x \in \Omega .
$$

Then $\widetilde{u}_{\nu} \in H^{2}(\Omega)$ and $\widetilde{u}_{\nu} \rightarrow s 1_{E}$ in $L^{2}(\Omega)$ as $\nu \rightarrow 0^{+}$. Using the coarea formula, we find

$$
\begin{aligned}
F_{\nu}\left(\widetilde{u}_{\nu}, A\right)= & \frac{1}{2} \quad \int_{A \cap V_{\eta \nu}}\left[\nu^{3}\left|u_{\nu}^{\prime \prime}(d)(\nabla d \otimes \nabla d)+u_{\nu}^{\prime}(d) \nabla^{2} d\right|^{2}+\frac{1}{\nu \phi(1 / \nu)} \phi\left(\left|u_{\nu}^{\prime}(d) \nabla d\right|\right)\right] d x \\
\leq & \frac{1}{2} \quad \int_{0}^{\eta \nu} \int_{\{x \in A: d(x)=t\}}\left[\nu^{3}\left(u_{\nu}^{\prime \prime}(t)\right)^{2}+2 \nu^{3}\left|u_{\nu}^{\prime \prime}(t)\left\|u_{\nu}^{\prime}(t) \mid+\nu^{3}\left(u_{\nu}^{\prime}(t)\right)^{2}\right\| \nabla^{2} d \|_{\infty}^{2}\right.\right. \\
& \left.\quad+\frac{1}{\nu \phi(1 / \nu)} \phi\left(\left|u_{\nu}^{\prime}(t)\right|\right)\right] d \mathcal{H}^{n-1} d t \\
=\frac{1}{2} & \int_{0}^{\eta \nu}\left(\nu^{3}\left(u_{\nu}^{\prime \prime}(t)\right)^{2}+\frac{1}{\nu \phi(1 / \nu)} \phi\left(\left|u_{\nu}^{\prime}(t)\right|\right)\right) \mathcal{H}^{n-1}(\{x \in A: d(x)=t\}) d t \\
& +\nu^{3} \int_{0}^{\eta \nu}\left(\left|u_{\nu}^{\prime \prime}(t)\right|\left|u_{\nu}^{\prime}(t)\right|+\frac{1}{2}\left(u_{\nu}^{\prime}(t)\right)^{2}\left\|\nabla^{2} d\right\|_{\infty}^{2}\right) \mathcal{H}^{n-1}(\{x \in A: d(x)=t\}) d t .
\end{aligned}
$$

We now claim that

$$
F_{\nu}\left(\widetilde{u}_{\nu}, A\right) \leq \sup _{t \in(0, \eta \nu)}\left[F_{\nu}\left(u_{\nu},(0, \eta \nu)\right) \mathcal{H}^{n-1}(\{x \in A: d(x)=t\})\right]+o(\nu) .
$$

Indeed, since $\lim _{\nu \rightarrow 0^{+}} F_{\nu}\left(u_{\nu},(-\delta, \delta)\right)<+\infty$, we have

$$
\sup _{\nu} \nu^{3} \int_{-\delta}^{\delta}\left(u_{\nu}^{\prime \prime}\right)^{2} d t<+\infty
$$

and by definition of $u_{\nu}$,

$$
\int_{0}^{\eta \nu}\left(u_{\nu}^{\prime}\right)^{2} d t=\frac{1}{\nu} \int_{0}^{\eta}\left(\psi^{\prime}\right)^{2} d t
$$


Thus by Cauchy-Schwarz's inequality,

$$
\nu^{3} \int_{0}^{\eta \nu}\left|u_{\nu}^{\prime \prime}\right|\left|u_{\nu}^{\prime}\right| d t \leq \nu\left(\int_{0}^{\eta \nu} \nu^{3}\left(u_{\nu}^{\prime \prime}\right)^{2} d t\right)^{\frac{1}{2}}\left(\int_{0}^{\eta}\left(\psi^{\prime}\right)^{2} d t\right)^{\frac{1}{2}}=O(\nu) .
$$

We can similarly bound the term $\nu^{3} \int_{0}^{\eta \nu}\left(u_{\nu}^{\prime}\right)^{2} d t\left\|\nabla^{2} d\right\|_{\infty}^{2}$. Finally since $\partial E$ is smooth,

$$
\lim _{t \rightarrow 0^{+}} \mathcal{H}^{n-1}(\{x \in A: d(x)=t\})=\mathcal{H}^{n-1}(A \cap \partial E) .
$$

Therefore, using the one-dimensional result in Section 3, we obtain

$$
\limsup _{\nu \rightarrow 0^{+}} F_{\nu}\left(\widetilde{u}_{\nu}, A\right) \leq \sigma_{a}|s|^{\frac{2+a}{4-a}} \mathcal{H}^{n-1}(A \cap \partial E)+b .
$$

Letting $b \rightarrow 0^{+}$we obtain the thesis.

We can now prove the $\Gamma$-limsup, following the proof of [4, Prop 3.6].

Theorem 5.5 (Г-limsup). We have

$$
\Gamma\left(L^{2}(A)\right)-\limsup _{\nu \rightarrow 0^{+}} F_{\nu}(\cdot, A) \leq \mathcal{F}(\cdot, A), \quad A \in \mathcal{A}(\Omega) .
$$

Proof. Thanks to the density Lemma 4.1, it is enough to prove the $\Gamma$-limsup inequality on those functions having a jump set contained in a finite union of facets of polytopes. Accordingly, we take

$$
u=\sum_{i=1}^{k} s_{i} 1_{C_{i}},
$$

where $k \in \mathbb{N}, s_{i} \in \mathbb{R}$ and $C_{i} \subseteq A$ are closed polytopes with pairwise empty intersection of their interior parts. We can further assume that all $C_{i}$ are convex. Set

$$
C_{k+1}:=A \backslash \bigcup_{i=1}^{k} C_{i}
$$

and

$$
\mathcal{I}:=\left\{(i, j): i, j \in\{i, \ldots, k+1\}, i<j, \mathcal{H}^{n-1}\left(C_{i} \cap C_{j}\right) \neq 0\right\}
$$

For $\delta>0$ let

$$
V^{\delta}:=\left\{x \in A: \operatorname{dist}\left(x, \bigcup_{i=1}^{k} \partial C_{i}\right)<\delta\right\}
$$

and

$$
V_{i j}^{\delta}:=\left\{x \in A: \operatorname{dist}\left(x, \partial C_{i} \cap \partial C_{j}\right)<\delta\right\}, \quad(i, j) \in \mathcal{I} .
$$

For any $(i, j) \in \mathcal{I}$ choose functions $g_{i j} \in \mathcal{C}_{c}^{\infty}\left(\mathbb{R}^{n}\right)$ such that

$$
g_{i j}=1 \text { on } V_{i j}^{\delta}, \quad g_{i j}=0 \text { on } A \backslash V_{i j}^{2 \delta},
$$

and

$$
\left\|\nabla g_{i j}\right\|_{\infty} \leq \frac{c}{\delta}, \quad\left\|\nabla^{2} g_{i j}\right\|_{\infty} \leq \frac{c}{\delta^{2}}
$$


where $c>0$ is a constant independent of $\delta, i$ and $j$. We now let

$$
h_{i j}:=g_{i j}\left(\sum_{(l, m) \in \mathcal{I}} g_{l m}+\prod_{(l, m) \in \mathcal{I}}\left(1-g_{l m}\right)\right)^{-1}
$$

and

$$
h_{0}:=\prod_{(l, m) \in \mathcal{I}}\left(1-g_{l m}\right)\left(\sum_{(l, m) \in \mathcal{I}} g_{l m}+\prod_{(l, m) \in \mathcal{I}}\left(1-g_{l m}\right)\right)^{-1} .
$$

These functions are defined so that

$$
h_{0}+\sum_{(i, j) \in \mathcal{I}} h_{i j}=1
$$

and

$$
h_{i j}=g_{i j} \text { on } V_{i j}^{2 \delta} \backslash \bigcup_{(l, m) \neq(i, j)} V_{l m}^{2 \delta} \quad \text { and } \quad h_{0}=1 \text { on } A \backslash V^{2 \delta} .
$$

It can be verified that

$$
\begin{aligned}
\left\|\nabla h_{i j}\right\|_{\infty} & \leq \frac{c}{\delta}, \quad\left\|\nabla h_{0}\right\|_{\infty} \leq \frac{c}{\delta}, \\
\left\|\nabla^{2} h_{i j}\right\|_{\infty} & \leq \frac{c}{\delta^{2}}, \quad\left\|\nabla^{2} h_{0}\right\|_{\infty} \leq \frac{c}{\delta^{2}} .
\end{aligned}
$$

Let $\left(u_{i j}^{\nu}\right) \subset H^{2}(\Omega)$ be the recovery sequence constructed as in Proposition 5.4 related to

$$
u_{i j}:= \begin{cases}s_{i}-s_{j} & \text { on } S_{i j}^{+} \\ 0 & \text { on } S_{i j}^{-}\end{cases}
$$

where $S_{i j}$ is the hyperplane containing $C_{i} \cap C_{j}$ and

$$
S_{i j}^{ \pm}:=\left\{x \in \Omega: x=y \pm t \nu_{i j}, y \in S_{i j}, t \in \mathbb{R}^{+}\right\},
$$

with $\nu_{i j}$ the unit internal normal to $C_{i}$ on $C_{i} \cap C_{j}$. We fix then $\delta=\eta \nu$ where $\eta$ is given by the one-dimensional profile. Let also

$$
u_{\nu}:=h_{0} u+\sum_{(i, j) \in \mathcal{I}} h_{i j}\left(u_{i j}^{\nu}+s_{j}\right)
$$

Then $u_{\nu} \in H^{2}(\Omega)$ and $u_{\nu} \rightarrow u$ in $L^{2}(\Omega)$ as $\nu \rightarrow 0^{+}$. Now on $V_{i j}^{2 \delta} \backslash \bigcup_{(l, m) \neq(i, j)} V_{l m}^{2 \delta}, u_{\nu}=$ $\left(1-g_{i j}\right) u+g_{i j}\left(u_{i j}^{\nu}+s_{j}\right)$ and since on $V_{i j}^{2 \delta} \backslash V_{i j}^{\delta}, u_{i j}^{\nu}=u_{i j}$, we then have on this set $u_{\nu}=u$ and $\nabla u_{\nu}=0$. Thus

$$
F_{\nu}\left(u_{\nu}, V_{i j}^{2 \delta} \backslash \bigcup_{(l, m) \neq(i, j)} V_{l m}^{2 \delta}\right)=F_{\nu}\left(u_{\nu}, V_{i j}^{\delta} \backslash \bigcup_{(l, m) \neq(i, j)} V_{l m}^{2 \delta}\right) \leq F_{\nu}\left(u_{i j}^{\nu}, A \cap V_{i j}^{\delta}\right) .
$$


On $V_{i j}^{2 \delta} \cap V_{l m}^{2 \delta}$, we have (remember that $\left|\nabla u_{i j}^{\nu}\right| \leq\left\|\psi^{\prime}\right\|_{\infty} / \nu$ )

$$
\left|\nabla u_{\nu}\right| \leq|u|\left|\nabla h_{0}\right|+\sum_{(i, j) \in \mathcal{I}}\left(\left|\nabla h_{i j}\right|\left|s_{j}\right|+\left|\nabla u_{i j}^{\nu}\right|\right) \leq \frac{C}{\nu},
$$

for a suitable constant $C>0$ independent of $\nu$. Since $\mathcal{H}^{n-2}\left(C_{i} \cap C_{j} \cap C_{l} \cap C_{m}\right)<+\infty$ (where $\mathcal{H}^{n-2}$ is the $(n-2)$-dimensional Hausdorff measure) we have $\left|V_{i j}^{2 \delta} \cap V_{l m}^{2 \delta}\right|=O\left(\nu^{2}\right)$ and thus

$$
\int_{V_{i j}^{2 \delta} \cap V_{l m}^{2 \delta}} \frac{1}{\nu \phi(1 / \nu)} \phi\left(\left|\nabla u_{\nu}\right|\right) d x \leq C \nu \frac{\phi(C / \nu)}{\phi(1 / \nu)} .
$$

As $\lim _{\nu \rightarrow 0^{+}} \frac{\phi(C / \nu)}{\phi(1 / \nu)}=C^{a}$, this shows that

$$
\lim _{\nu \rightarrow 0^{+}} \int_{V_{i j}^{2 \delta} \cap V_{l m}^{2 \delta}} \frac{1}{\nu \phi(1 / \nu)} \phi\left(\left|\nabla u_{\nu}\right|\right) d x=0 .
$$

Similarly,

and therefore

$$
\lim _{\nu \rightarrow 0^{+}} \int_{V_{i j}^{2 \delta} \cap V_{l m}^{2 \delta}} \nu^{3}\left|\nabla^{2} u_{\nu}\right|^{2} d x=0
$$

$$
\lim _{\nu \rightarrow 0^{+}} F_{\nu}\left(u_{\nu}, V_{i j}^{2 \delta} \cap V_{l m}^{2 \delta}\right)=0 .
$$

Eventually on $A \backslash V^{2 \delta}, u_{\nu}=u$ and $\nabla u=0$, thus

$$
F_{\nu}\left(u_{\nu}, A \backslash V^{2 \delta}\right)=0 .
$$

Putting together (5.11), (5.12) and (5.13) we find by Proposition 5.4,

$$
\begin{aligned}
\lim _{\nu \rightarrow 0^{+}} F_{\nu}\left(u_{\nu}, A\right) & \leq \sum_{(i, j) \in \mathcal{I}^{\nu \rightarrow 0^{+}}} \lim _{\nu}\left(u_{\nu}, V_{i j}^{2 \delta} \bigcup_{(l, m) \neq(i, j)} V_{l m}^{2 \delta}\right) \\
& \leq \sum_{(i, j) \in \mathcal{I}^{\nu \rightarrow 0^{+}}} F_{\nu}\left(u_{\nu}^{i j}, V_{i j}^{\delta}\right) \\
& =\sum_{(i, j) \in \mathcal{I}^{\nu \rightarrow 0^{+}}} \lim _{\nu}\left(u_{\nu}^{i j}, A\right) \\
& =\sigma_{a} \sum_{(i, j) \in \mathcal{I}}\left|s_{i}-s_{j}\right|^{\frac{2+a}{4-a}} \mathcal{H}^{n-1}\left(A \cap C_{i} \cap C_{j}\right) \\
& =\mathcal{F}(u, A) .
\end{aligned}
$$

Remark 5.6. Differently with respect to the results of $[4,39]$ (which rely on the density theorem of [24]) using Lemma 4.1 we obtain a full $\Gamma$-convergence result. Moreover, we have compactness in $B V$ for the $n$-dimensional problem which is a missing feature in most of the papers tackling similar problems such as $[4,39]$.

We conclude by mentioning that our theorems are a first step toward a general approximation theorem in $S B V$ (see the related papers $[23,24,2,4]$ ) for free discontinuity functionals having, in general, also a bulk term. 


\section{Acknowledgments}

The first two authors thank the hospitality of the Mathematisches Forschungsinstitut Oberwolfach, which gave them the opportunity to start this research.

\section{References}

[1] G. Alberti, S. MÜller: New approach to variational problems with multiple scales, Comm. Pure Appl. Math. 54 (2001), 761-825.

[2] R. Alicandro, A. Braides, M.S. Gelli: Free-discontinuity problems generated by singular perturbations, Proc. Roy. Soc. Edinburgh Sect. A 128 (1998), 1115-1129.

[3] R. Alicandro, A. Braides, J. Shah: Free-discontinuity problems via functionals involving the $L^{1}$-norm of the gradient and their approximations, Interfaces Free Bound. 1:1 (1999), 17-37.

[4] R. Alicandro, M.S. Gelli: Free-discontinuity problems generated by singular perturbation: the n-dimensional case, Proc. Roy. Soc. Edinburgh Sect. A 130 (2000), 449-469.

[5] L. Ambrosio, N. Dancer: Calculus of variations and partial differential equations. Topics on geometrical evolution problems and degree theory, Papers from the Summer School held in Pisa, September 1996, Edited by G. Buttazzo, A. Marino and M. K. V. Murthy, Springer-Verlag (Berlin), 2000.

[6] L. Ambrosio, N. Fusco, D. Pallara: Functions of Bounded Variation and Free Discontinuity Problems, Clarendon Press (Oxford), 2000.

[7] L. Ambrosio, A. Lemenant, G. Royer-Carfagni: A variational model for plastic slip and its regularization via Gamma-convergence, to appear in J. of Elasticity.

[8] L. Ambrosio, V.M. Tortorelli: On the approximation of free discontinuity problems, Boll. Unione Mat. Ital. VII Ser. B 6 (1992), 105-123.

[9] G. Bellettini, G. Fusco: The $\Gamma$-limit and the related gradient flow for singular perturbation functionals of Perona-Malik type, Trans. Amer. Math. Soc. 360 (2008), 4929-4987.

[10] G. Bellettini, M. Novaga, E. Paolini: Global solutions to the gradient flow equation of a nonconvex functional, SIAM J. Math. Anal., 37 (2006), 1657-1687.

[11] G. Bellettini, M. Novaga, M. Paolini, C. Tornese: Convergence of discrete schemes for the Perona-Malik equation, J. Differential Equations, 245 (2008), 892-924.

[12] G. Bellettini, M. Novaga, M. Paolini, C. Tornese: Classification of the equilibria and $\Gamma$-convergence for the semi-discrete Perona-Malik functional, Calcolo, 46 (2009), $221-243$.

[13] G. Bouchitté, C. Dubs, P. Seppecher: Transitions de phases avec un potentiel dégénéré à l'infini, application à l'équilibre de petites gouttes, C. R. Acad. Sci. Paris Sér. I Math. 323:9 (1996), 1103-1108. 
[14] B. Bourdin, G. Francfort, J.-J. Marigo: The variational approach to fracture, J. of Elasticity 91:1-3 (2008), 5-148.

[15] A. Braides: Approximation of Free-Discontinuity Problems, Lecture Notes in Mathematics, 1694. Springer-Verlag (Berlin), 1998.

[16] A. Braides: $\Gamma$-convergence for beginners, Oxford Lecture Series in Mathematics and its Applications, 22. Oxford University Press (Oxford), 2002.

[17] A. Chambolle: An approximation result for special functions with bounded deformation, J. Math. Pures Appl. 9:83 (2004), 929-954.

[18] A. Chambolle: Addendum to: "An approximation result for special functions with bounded deformation" [J. Math. Pures Appl. (9) 83 (2004), 929-954] J. Math. Pures Appl. 9:84 (2005), 137-145.

[19] A. Chambolle, A. Giacomini, M. Ponsiglione: Piecewise rigidity, J. Funct. Anal. 244 (2007), 134-153.

[20] M. Colombo, M. Gobbino: Slow time behavior of the semidiscrete Perona-Malik scheme in dimension one, to appear in SIAM J. Math. Anal.

[21] S. Conti, I. Fonseca, G. Leoni: A $\Gamma$-convergence result for the two-gradient theory of phase transitions, Comm. Pure Appl. Math. 55 (2002), no. 7, 857-936.

[22] S. Conti, B. Schweizer: Rigidity and gamma convergence for solid-solid phase transitions with SO(2) invariance, Comm. Pure Appl. Math. 59 (2006), 830-868.

[23] G. Cortesani: Sequences of non-local functionals which approximate free discontinuity problems, Arch. Ration. Mech. Anal. 144 (1998), 357-402.

[24] G. Cortesani, R. Toader: A density result in $S B V$ with respect to non isotropic energies, Nonlinear Anal. 38 (1999), Ser. B: Real World Appl., 585-604.

[25] G. Dal Maso: An Introduction to $\Gamma$-convergence, Birkhäuser (Boston), 1993.

[26] G. Dal Maso, F. Iurlano: Fracture models as $\Gamma$-limits of damage models, Comm. Pure Appl. Anal. 12:4 (2013), 1657-1686.

[27] E. De Giongi: Congetture riguardanti alcuni problemi di evoluzione, Duke Math. J. 81 (1995), 255-268.

[28] S. Esedoglu: An analysis of the Perona-Malik scheme, Comm. Pure Appl. Math. 54 (2001), no. 12, 1442-1487.

[29] L. C. Evans, G. Gariepy, Measure Theory and Fine Properties of Functions, Studies in Advanced Mathematics, CRC Press, 1992.

[30] I. Fonseca, C. Mantegazza: Second order singular perturbation models for phase transitions, SIAM J. Math. Anal. 31 (2000), 1121-1143

[31] G. A. Francfort, J.J. Marigo: Revisiting brittle fracture as an energy minimization problem J. Mech. Phys. Solids 46 (1998), no. 8, 1319-1342. 
[32] E. Giusti: Minimal surfaces and functions of bounded variation, Monographs in Mathematics 80, Birkhäuser (Boston), 1984.

[33] M. Ghisi, M. GobBino: Gradient estimates for the Perona-Malik equation, Math. Ann., 337 (2007), 557-590.

[34] M. GobBino: Finite difference approximation of the Mumford-Shah functional. Comm. Pure Appl. Math. 51 (1998), 197-228.

[35] M. Gobbino: Entire solutions of the one-dimensional Perona-Malik equation, Comm. Partial Differential Equations 32 (2007), 4-6, 719-743.

[36] M. Gobbino, M. G. Mora: Finite-difference approximation of free-discontinuity problems Proc. Roy. Soc. Edinburgh Sect. A 131 (2001), no. 3, 567-595.

[37] B. Kawohl, N. Kutev: Maximum and comparison principle for one-dimensional anisotropic diffusion, Math. Ann. 311 (1998), 107-123.

[38] S. Kichenassamy: The Perona-Malik paradox, SIAM J. Appl. Math. 57 (2001), 13281342 .

[39] M. Morini: Sequences of singularly perturbed functionals generating free-discontinuity problems, SIAM J. Math. Anal. 35 (2003), 759-805.

[40] D. Mumford, J. Shah : Optimal approximations by piecewise smooth functions and associated variational problems, Comm. Pure Appl. Math. 42 (1989), no. 5, 577-685.

[41] E. Oudet, F. Santambrogio: A Modica-Mortola approximation for branched transport and applications, Arch. Ration. Mech. Anal. 201 (2011), no. 1, 115-142.

[42] P. Perona, J. Malik: Scale space and edge detection using anisotropic diffusion, IEEE Trans. Pattern Anal. Mach. Intell. 12 (1990), 629-639.

[43] E. SAndier, S. Serfaty: Г-convergence of gradient flows with applications to GinzburgLandau, Comm. Pure Appl. Math. 57 (2004), 1627-1672.

[44] M. Slemrod: Dynamics of measure valued solutions to a backward-forward heat equation, J. Dynam. Differential Equations, 3 (1991), 1-28. 